\title{
Soft gluon resummation in the infrared region and the Froissart bound
}

\author{
Giulia Pancheri*† \\ INFN Frascati National Laboratories, Via E. Fermi 40, I00044 Frascati, Italy \\ E-mail: pancherielnf.infn.it
}

\begin{abstract}
We describe the taming effect induced by soft gluon $k_{t}$-resummation on the rapid rise of QCD mini-jet contributions to the total cross-sections. This results from an eikonal model in which the rise of the total cross-section is due to mini-jet contribution. We perform the calculation with current Parton Density Functions (PDFs). The impact parameter distribution we use is obtained as the Fourier transform of the resummed $k_{t}$-distribution of soft gluons emitted from the initial state during the collision. The emission, which is energy dependent, destroys the initial collinearity of partons. In this model, the strong power-like rise due to the increasing number of low-x gluon collisions is tamed by the acollinearity induced by soft gluon kt-resummation down to zero gluon momenta. It explicitly links a singular soft gluon coupling in the infrared region to the behaviour dictated by the Froissart bound for the total cross-section. The model describes well both proton and photon processes at present accelerator energies and gives predictions in the TeV range. For photons the model predictions agree with fits by Block and Halzen based on Finite Energy Sum Rules (FESR) at low energy and an asymptotic behaviour consistent with the Froissart bound.
\end{abstract}

XVIII International Workshop on Deep-Inelastic Scattering and Related Subjects, DIS 2010 April 19-23, 2010

Firenze, Italy

\footnotetext{
* Speaker.

$\dagger$ Visitor at CTP, MIT, Cambridge, MA
} 


\section{Our model}

Our model for the total cross-section [1] starts with the simplified eikonal formulation

$$
\sigma_{\text {total }} \approx 2 \int d^{2} \mathbf{b}\left[1-e^{-\bar{n}(b, s) / 2}\right]
$$

where the real part of the scattering amplitude has been approximated to zero and

$$
\bar{n}(b, s)=\bar{n}_{s o f t}(b, s)+\bar{n}_{Q C D}(b, s)=A_{s o f t}(b, s) \sigma_{s o f t}(s)+A_{B N}(b, s) \sigma_{\text {jet }}\left(s, p_{t m i n}\right)
$$

The mini-jet cross-section, $\sigma_{\text {jet }}\left(s, p_{\text {tmin }}\right)$, is calculated using parton-parton cross-sections and DGLAP evoluted PDFs, integrating over all parton final state momenta such that $p_{t} \geq p_{t \min }$. $A_{B N}$ is the impact parameter distribution for the mini-jet processes which we describe in the next section and which leads to a cut-off at large $b$-values with a behaviour stronger than an exponential [2]. The soft part of the cross-section, which dominates up to $\sqrt{s} \sim 10 \mathrm{GeV}$ is parametrized as described in [1].

We shall refer to this model as Bloch-Nordsieck (BN) model because of the indispensible role played in our model by the resummation of soft massless quanta in gauge theories. We were inspired to build our model by the classic work of Bloch and Nordsieck [3] in electrodynamics, where they first pointed out that only the emission of an infinite number of soft photons can lead to a finite result.

In our model, such a soft gluon $k_{t}$-resummation and its implementation down into the gluon infrared momentum region, constitute the mechanisms through which the fast initial rise of all the total cross-sections is transformed into a smooth logarithmic rise, consistent with the Froissart bound. We have obtained results for proton and photon total cross-sections as shown in Fig. 1 from [4] . In Fig. 1, left panel, we show a compilation of proton and photon total cross-section data, where photon data have been multiplied by an ad hoc factor proportional to $\alpha_{Q E D}$. Our model description for $p p$ and $\bar{p} p$ is indicated by the band and by the full line.

When dealing with photons, the eikonal model we have used needs to be modified so as to take into account the transition of the photon into a hadron when the photon interacts with matter. Indicating the probability for this transition as $P_{\text {had }}$, we follow the model proposed originally in [6] and write

$$
\sigma_{\text {total }}^{\gamma p}=2 P_{\text {had }} \int d^{2} \mathbf{b}\left[1-e^{-\bar{n}^{\gamma p}(b, s) / 2}\right]
$$

with the average number of collisions to be calculated using again minijets and the known PDFs for the photon. With $P_{\text {had }}=1 / 240$ (through Vector Meson Dominance arguments)), for $\gamma p$ we find [4] that the cross-section remains proportional to the proton cross-sections up to present accelerator energies (yellow band in right panel of Fig. 1), but it becomes quite a bit larger at higher energies. On the other hand, our results agree with the results of Block and Halzen [5], who combine a low energy fit from FESR with a maximally allowed $\ln ^{2} s$ behaviour at high energy.

\section{The impact parameter distribution in the $\mathrm{BN}$ model}

The impact parameter distribution $A(b)$ has a simple semi-classical interpretation as the convolution of form factors of the colliding particles [7]. However, in the energy region where mini-jets 

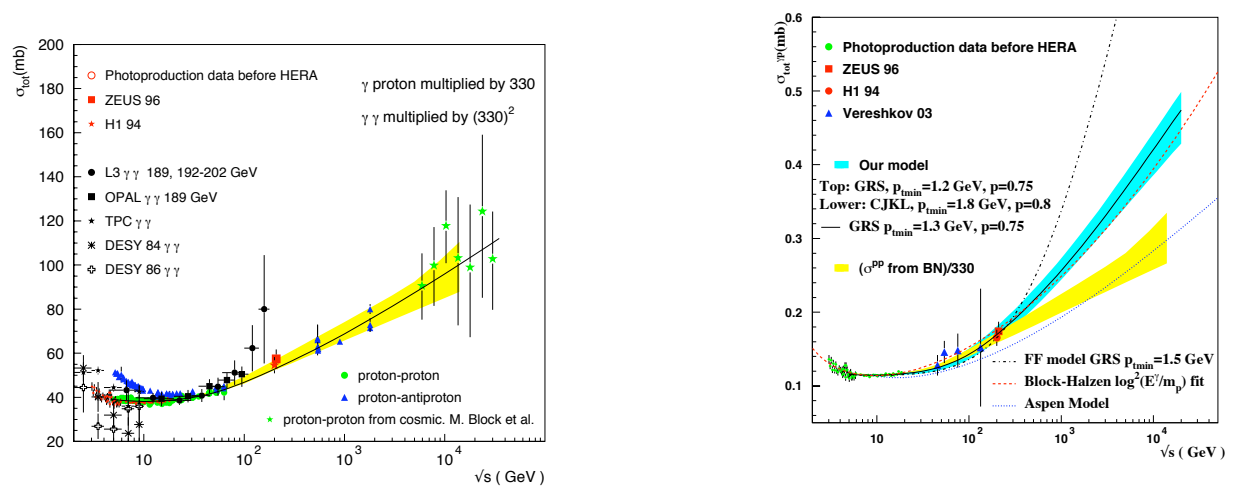

Figure 1: From [4]: at left is a compilation of data for $p p, p \bar{p}, \gamma p$ and $\gamma \gamma$ with the yellow band showing our predictions. Photon data have been multiplied by an ad hoc factor proportional to $\alpha_{Q E D}$. At right is the $\gamma p$ total cross-section, with the yellow band obtained multiplying the $p p$ band of the left panel by a numerical factor, the blue line is our model for $\gamma p$ and the Bloch-Halzen line is from [5].

start playing a role, the form factor expression is inadequate. Our proposal is that the impact parameter distribution $A_{B N}(b, s)$ in parton-parton scattering be derived from initial state soft gluon $k_{t}$-resummation $[8,9]$. It is given by the normalized Fourier transform of the $k_{t}$-resummed expression for soft gluon initial state emission in parton-parton collision. Because the total cross-section is dominated by large impact parameter values, we have revisited this distribution, extending the integration over single soft gluon momenta into the infrared region, as discussed in [10] and described at length for protons [1] and recently for photons [4].

The expression we use is

$$
A_{B N}(b, s)=\frac{e^{-h\left(b, q_{\max }\right)}}{\int d^{2} \vec{b} e^{-h\left(b, q_{\max }\right)}}=\frac{e^{-\frac{16}{3 \pi} \int_{0}^{q \max } \frac{d k_{t} \alpha_{e f f}\left(k_{t}\right)}{k_{t}} \ln \left(\frac{2 q_{\max }}{k_{t}}\right)\left[1-J_{0}\left(b, k_{t}\right)\right]}}{\int d^{2} \vec{b} e^{-h\left(b, q_{\max }\right)}}
$$

where the function $h\left(b, q_{\max }\right)$ is obtained from soft gluon resummation techniques and has a logarithmic energy dependence through the scale $q_{\max }$, which is proportional to $p_{\text {tmin }}$. To obtain the taming effect which will change the fast rise of mini-jets in a logarithmic behaviour a' la Froissart, it is necessary to extend resummation down to single soft gluon momenta below $\Lambda_{Q C D}$. To do this, a model for the infrared gluon distribution has to be introduced, as explained in [2]. With an effective coupling $\alpha_{e f f}\left(k_{t}\right) \sim k_{t}^{-2 p}$ for the single soft gluon transverse momentum in the region $0 \leq k_{t} \leq \Lambda_{Q C D}$ and $1 / 2<p<1$, we have shown in [2] how this expression for $A_{B N}(b, s)$ introduces a strong cut-off in $b$-space and changes the violent rise of mini-jets into a smooth behavior in the total cross-section. Namely, we found that $\sigma_{t o t} \sim(\ln s)^{1 / p}$, a behaviour consistent with the limitations imposed by the Froissart bound.

For the low energy region, where minijets do not yet play a role, we use the parametrization proposed in [1] for $A_{s o f t}(b, s)$, which was also inspired by the soft gluon resummation model. 
To compare our result for $\bar{n}^{p p}(b, s)$ at LHC with other models, and in particular with models which use the form factors both at low and at high energy, we can define an average $b$-distribution such that

$$
A_{\text {mean }}^{p p}(b, s)=\frac{\bar{n}(b, s)}{\sigma_{\text {soft }}^{p p}(s)+\sigma_{\text {jet }}^{p p}\left(s, p_{\text {tmin }}\right)}
$$

We show the energy dependence of $q_{\max }$ in Fig. 2 and $A(b)$ from our $k_{t}$-resummation model in Figs. 3,4,5,6. In all the figures, we have chosen $p_{\text {tmin }}=1.15 \mathrm{GeV}$, used the GRV densities and $p=0.75$, unless indicated. In these figures we compare our model for $A(b, s)$ with the Form Factor model expression

$$
A_{F F}(b)=\frac{1}{(2 \pi)^{2}} \int d^{2} \vec{q} e^{i \vec{q} \cdot \vec{b}}\left[\mathscr{F}_{p}(q)\right]^{2}=\frac{1}{(2 \pi)^{2}} \int d^{2} \vec{q} e^{i \vec{q} \cdot \vec{b}} \frac{1}{\left[1+(q / v)^{2}\right]^{4}}
$$

where $\mathscr{F}_{p}$ is the proton form factor with $v^{2}=0.71 \mathrm{GeV}^{2}$.

\section{Acknowledgments}

R.G. acknowledges support from the Department of Science and Technology, India under Grant No. SR/S2/JCB-64/2007, under the J.C. Bose Fellowship scheme. Work partially supported by the Spanish MEC (FPA2006-05294 and FPA2008-04158-E/INFN) and by Junta de Andalucía (FQM 101). G.P gratefully acknowledges the hospitality of the MIT Center for Theoretical Physics. YS is also an Emeritus Professor at Northeastern University Boston MASS and thanks the physics department for its hospitality.

\section{References}

[1] R.M. Godbole, A. Grau, G. Pancheri, Y.N. Srivastava, Soft gluon radiation and energy dependence of total hadronic cross-sections, Phys. Rev. D 72 (2005) 076001 [hep-ph/ 0408355$].$

[2] A. Grau, R.M. Godbole, G. Pancheri, Y.N. Srivastava, Soft Gluon k(t)-Resummation and the Froissart bound, Phys. Lett. B 682 (2009) 55 [hep-ph/ 0908.1426$].$

[3] F. Bloch, A. Nordsieck, Note on the Radiation Field of the electron, Phys. Rev. 52 (1937) 54.

[4] R.M. Godbole, A. Grau, G. Pancheri, Y.N. Srivastava, Total photoproduction cross-section at very high energy, EPJC 63 (2009) 69 [hep-ph/ 0812 . 1065].

[5] M. Block, F. Halzen, New evidence for the saturation of the Froissart bound Phys. Rev. D72 (2005), 036006, Erratum-ibid.D72 (2005) 039902, [hep-ph / 0506031$].$

[6] R.S. Fletcher, T.K. Gaisser, F. Halzen, Reexamining the jet contribution to the photoproduction cross-section, Phys.Rev. D45 (1992) 377, Erratum-ibid.D45(1992) 3279.

[7] L. Durand, H. Pi, Semihard QCD and high Energy $p$ p and anti-p p scattering Phys. Rev. D40 (1989) 1436.

[8] Yu. Dokshitzer, D. Diakonov, S.I. Troyan,On the Transverse Momentum Distribution of Massive Lepton Pairs, Phys. Lett. B79 (1978) 269.

[9] G. Parisi and R. Petronzio, Small Transverse Momentum Distributions in Hard Processes, Nucl.Phys. B154 (1979) 427.

[10] A. Corsetti, A. Grau, G. Pancheri, Y.N. Srivastava, Bloch-Nordsieck summation and partonic distributions in impact parameter space, Phys. Lett. B382 (1006) 282[hep-ph/9605314]. 


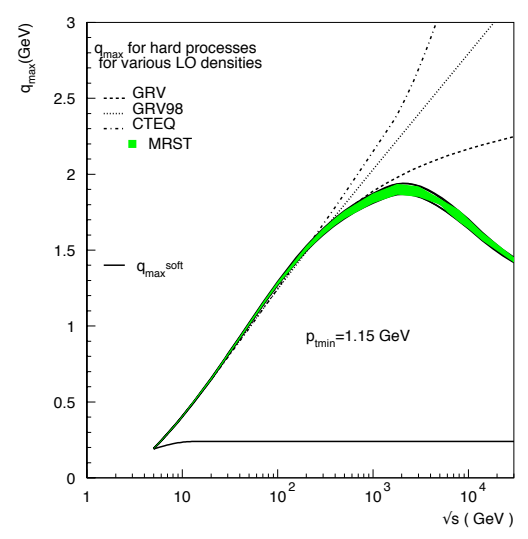

Figure 2: The maximum single soft gluon transverse momentum $p p$ or $\bar{p} p$ scattering for different PDFs.

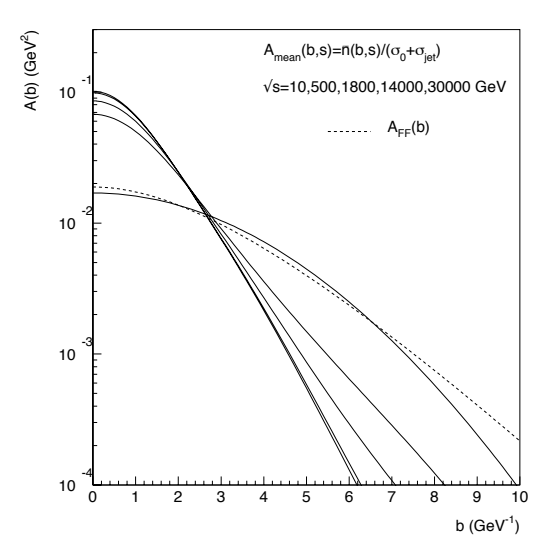

Figure 4: The model impact parameter distribution for protons at various energies.

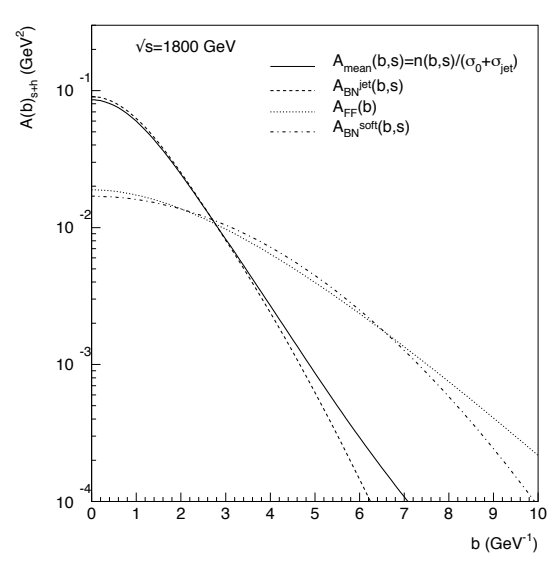

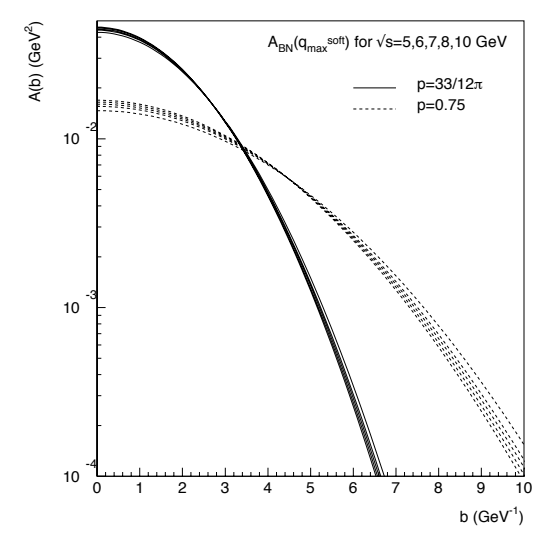

Figure 3: The model impact parameter distribution at various low-energy values for two values of the parameter $p$.

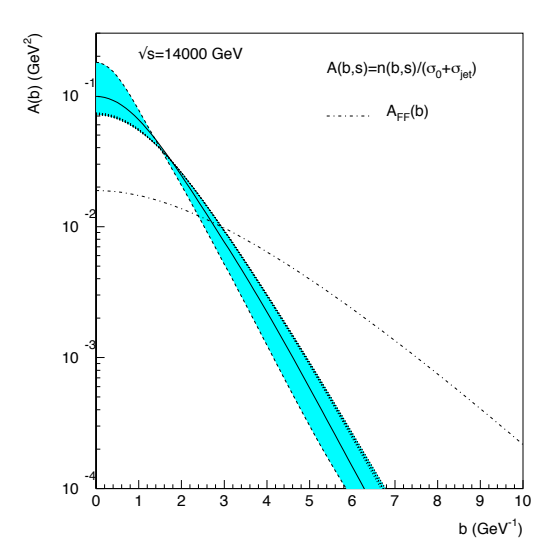

Figure 5: The model impact parameter distribution at LHC for different PDFs.

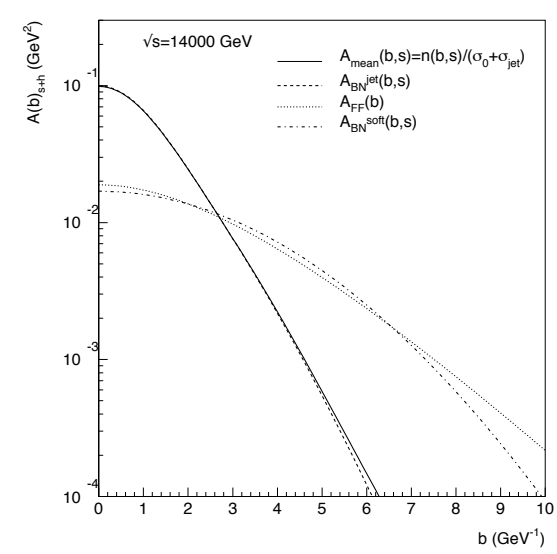

Figure 6: Different impact parameter distributions for the Tevatron and the LHC energies. 